\title{
Indicadores del Observatorio de Economía y Sociedad OBSERVES del IIESES-UV. Interpretación de resultados
}

\section{Indicators of the Observatory of Economy and Society OBSERVES of IIESES-UV. Interpretation of results}

\author{
Alberto García Leyva ${ }^{a}$ \\ a Observatorio de Economía y Sociedad, Instituto de Investigaciones y \\ Estudios Superiores Económicos y Sociales (IIESES), Universidad Veracruzana. \\ Contacto: albertogarcialeyva12@hotmail.com
}

Recibido: 18 de junio de 2020

Aceptado: 15 de octubre de 2020

RESUMEN: En el Observatorio de Economía y Sociedad (OBSERVES) del Instituto de Investigaciones y Estudios Superiores Económicos y Sociales (IIESES) y la Universidad Veracruzana (UV), se monitorean una serie de indicadores desarrollados y diseñados para conocer la realidad económica y social que envuelve a un individuo. Los indicadores expuestos en este trabajo de investigación, son la Sustracción Nominal Básica (SNB) y la Proporción Salarial Hídrica Doméstica (PSHD), los cuales se sigue su evolución a través del tiempo y sus resultados son publicados en la página web del observatorio, los indicadores con sus particularidades metodológicas, forman parte de una contribución a la sociedad en general interesada en el tema, con el objetivo de ayudar a entender problemáticas complejas resumidas en números de fácil comprensión para el público en general.

Palabras clave: Observatorio de Economía y Sociedad; indicadores; Sustracción Nominal Básica; Proporción Salarial Hídrica Doméstica; políticas públicas.

ABSTRACT: In the Observatory of Economy and Society (OBSERVES) of the Instituto de
Investigaciones y Estudios Superiores Económicos y Sociales (IIESES) and Universidad
Veracruzana (UV), a series of indicators developed and designed to know the economic and social
reality that involves An individual. The indicators presented in this research work are the Basic
Nominal Subtraction (SNB) and the Domestic Water Salary Ratio (PSHD), which follow its 
evolution over time and its results are published on the website of the observatory, the Indicators, with their methodological peculiarities, are part of a contribution to the general society interested in the subject, with the aim of helping to understand complex problems summarized in numbers that are easily understood by the general public.

Keywords: Economy and Society Observatory; Indicators; Basic Nominal Subtraction; Domestic Water Wage Ratio; Public Policies.

\footnotetext{
l Observatorio de Economía y Sociedad (OBSERVES) es un observatorio del Instituto $\longrightarrow$ de Investigaciones y Estudios Superiores Económicos y Sociales (IIESES), de la Universidad Veracruzana (UV), que monitorea una serie de indicadores a través del tiempo; uno de estos, desde finales del año 2017. Estos indicadores tienen una serie de características entre las que se encuentran ser metodologías originales, diseñadas y planeadas específicamente para seguirse en el observatorio.
}

La idea primordial de los indicadores de OBSERVES es capturar la problemática que se presenta en las condiciones económicas y sociales de los ciudadanos comunes, las adversidades que experimenta una persona promedio en México, especialmente en la entidad Veracruzana y en la ciudad de Xalapa; aunque los indicadores también se pueden calcular para otras ciudades de México, inclusive del mundo.

La manera en la que se publica los datos del seguimiento de estos indicadores es mediante boletines informativos, en donde se muestra el último resultado y una serie de gráficas comparativas entre los pasados periodos de análisis, éstos son publicados en la página web de OBSERVES (www.uv.mx/observes).

Este trabajo de investigación tiene el objetivo de mostrar una reseña de los indicadores; Sustracción Nominal Básica (SNB) y la Proporción Salarial Hídrica Doméstica (PSHD), y se mencionarán cuáles son sus posibles funciones, los supuestos en los que se basan y la contribución que representan.

\section{La SNB interpretación de resultados y eventos importantes}

La SNB es un indicador que mide el gasto que tiene una sola persona durante un solo día en materia de obtención de productos para una alimentación saludable, el salario mínimo vigente es comparado con dicho gasto; la diferencia entre estas dos cantidades se le llama Sustracción 
Nominal Básica, el resultado se expresa en pesos mexicanos nominales, se calcula para la ciudad de Xalapa en el estado de Veracruz y cumple con características de fácil compresión, adaptabilidad para otras zonas geográficas y comparabilidad, lo que se intenta medir versa sobre el ingreso mínimo que una persona puede tener y el gasto que representa tener una vida digna a través de una alimentación saludable, los productos seleccionados para costear el resultado previenen de una ejemplificación de un menú ideal saludable para consumo mexicano de la Secretaría de Salud de México y al gasto final se le denomina Gasto Diario Nutrimental (GDN), posteriormente se toma el salario mínimo y se le resta el GDN, dando como resultado la SNB (García, 2018a).

Los resultados son publicados en la página web de OBSERVES, a manera de boletines informativos, que están disponibles para ser visualizados de manera sencilla y de fácil interpretación. Su publicación data desde los últimos meses del 2017. Los boletines se presentan dos veces al mes, salvo en algunas ocasiones. En una primera parte, las fuentes de información primarias eran la Profeco, Procuraduría Federal del Consumidor y el SAT, Servicio de Administración Tributaría de la Secretaria de Hacienda y Crédito Público SHCP; pero posteriormente se utiliza información del Sistema Nacional de Información e Integración de Mercados SNIIM de la Secretaría de Economía e información de la Comisión Nacional de los Salarios Mínimos (OBSERVES, S.F. a).

El boletín número 53 del día 22 de mayo del 2020 de la Sustracción Nominal Básica (OBSERVES; García, 2020a), en una primera parte explica de manera general la fórmula de la SNB y las fuentes primarias que se consultan, seguido de una serie de enlaces a la metodología original, otros indicadores y a la página web de OBSERVES; en una segunda parte se muestran los últimos resultados que conforman el indicador. Para esta ocasión se presentó un GDN de \$81.36 con un salario mínimo vigente de \$123.22 y un resultado final de la SNB de \$41.85, resultados expuestos mencionando su respectiva fuente primaria, por último, en una tercera parte, se muestran unas graficas explicativas de la evolución del indicador. 


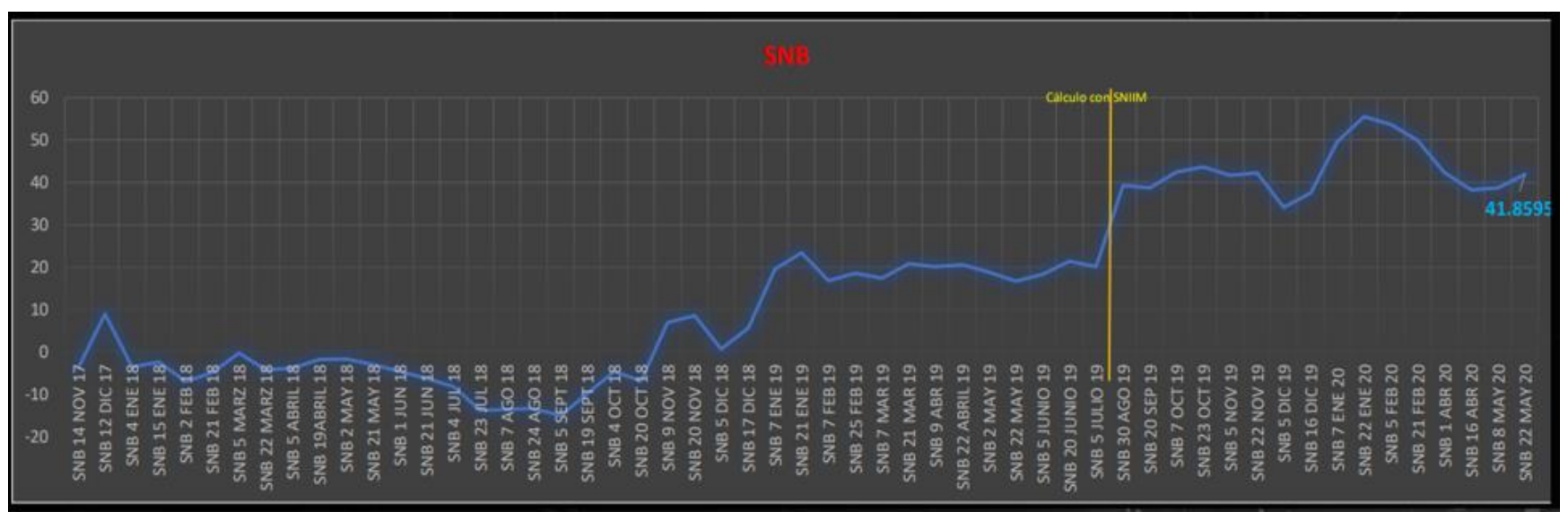

Figura 1. SNB noviembre 2017-mayo 2020. Fuente: Imagen capturada del Observatorio de Economía y Sociedad (OBSERVES); García. (2020a). Sustracción Nominal Básica (SNB). Boletín número 53 del día 22 de mayo de 2020. Autor: Alberto García Leyva. Disponible en: https://www.uv.mx/observes/files/2018/05/SNB-2020-53-1.pdf

En la figura 1, boletín número 53 de la SNB, se puede observar la evolución desde el mes de noviembre de 2017 a mayo de 2020, el cual presenta resultados mixtos. En una parte del periodo comprendido del 2018, se presentó una SNB negativa. Esto significaba que si una persona que ganaba el salario mínimo vigente de ese año, debía endeudarse para alimentarse sanamente con una cantidad cercana a los 15 pesos. Posteriormente, el indicador tiene resultados positivos motivados principalmente por los aumentos al salario mínimo, comportamiento que se puede observar en los inicios de año, en la gráfica presentada en el boletín, se señala el cambio de fuente primaria de consulta de precios, ya que se menciona que a partir de agosto del 2019 se calculó con el SNIIM.

Este tipo de información sirve típicamente para los diversos sectores de la sociedad, desde estudiantes, académicos a personas interesadas en el tema. También invita a calcular un gasto "personalizado" con diversos otros tipos de productos. Debido a la manera en la que se compone la fórmula de la SNB, se puede encontrar que ciertas políticas podrían influir en el comportamiento del indicador. Por una parte, de la Comisión Nacional de los salarios mínimos por el movimiento del salario mínimo y del Banco de México (BANXICO), que tiene la misión de controlar los niveles de inflación; la Comisión Nacional de los salarios mínimos cumple con la política de aumentarlo cada inicio de año, el BANXICO, por su parte, en los días en los que

se elabora esta investigación, junio del 2020, mantiene políticas monetarias expansivas, esto por la disminución de tasas de interés como consecuencia de la contingencia mundial del COVID-19, esta política de orden macroeconómico puede tener un impacto local en los precios que se vean reflejados en la SNB. 
Por la naturaleza del indicador de la SNB, es fehaciente que se siguen una serie de supuestos al momento de calcularlo: 1) las cantidades a consumir son un promedio general, ya que existen personas que por diversas condiciones de edad, estatura y físico comen más o menos que el promedio; 2) la elección de la presentación y los tipos de productos a calcular depende de la disponibilidad de información y también se trata de emular las elecciones variadas del consumidor, en el sentido de que, en un periodo de tiempo, elije una marca o presentación y en otro periodo opta por otro; 3) los precios que se toman son los publicados por las diversas fuentes e investigación de campo, podrían existir otros precios en otras partes como tiendas y mercados; 4) actualización de los precios en las fuentes primarias de información que no siempre se pueden dar al mismo tiempo que del cálculo de la SNB; 5) otros diversos supuestos que van cambiando dependiendo la temporalidad y contexto.

Por lo anterior, pueden existir diversas Sustracciones Nominales Básicas así que la SNB y sus resultados deben tomarse como tendencias y su comportamiento, si es positivo o negativo a través del tiempo, aunque el cálculo se arroje en pesos y centavos, lo correcto en la interpretación sería a través de su variación en la tendencia.

\section{La SNB y el contexto ante la pandemia del Covid-19}

Ante la complicada situación en la que se ve envuelto el mundo por la contingencia del COVID19, a la fecha de elaboración de esta investigación, el OBSERVES publicó un boletín especial abordando el tema del comportamiento de la SNB ante este contexto (OBSERVES; García, 2020b) en donde se menciona que el fenómeno mundial de la pandemia ha tenido repercusiones en la economía global y se muestran una serie de graficas en las que se señalan fechas importantes de la evolución de la pandemia, que pueden o no tener relación con el comportamiento de la SNB. La información para la construcción de este boletín especial se toma de SNIIM, Profeco, SHCP y BANXICO. 


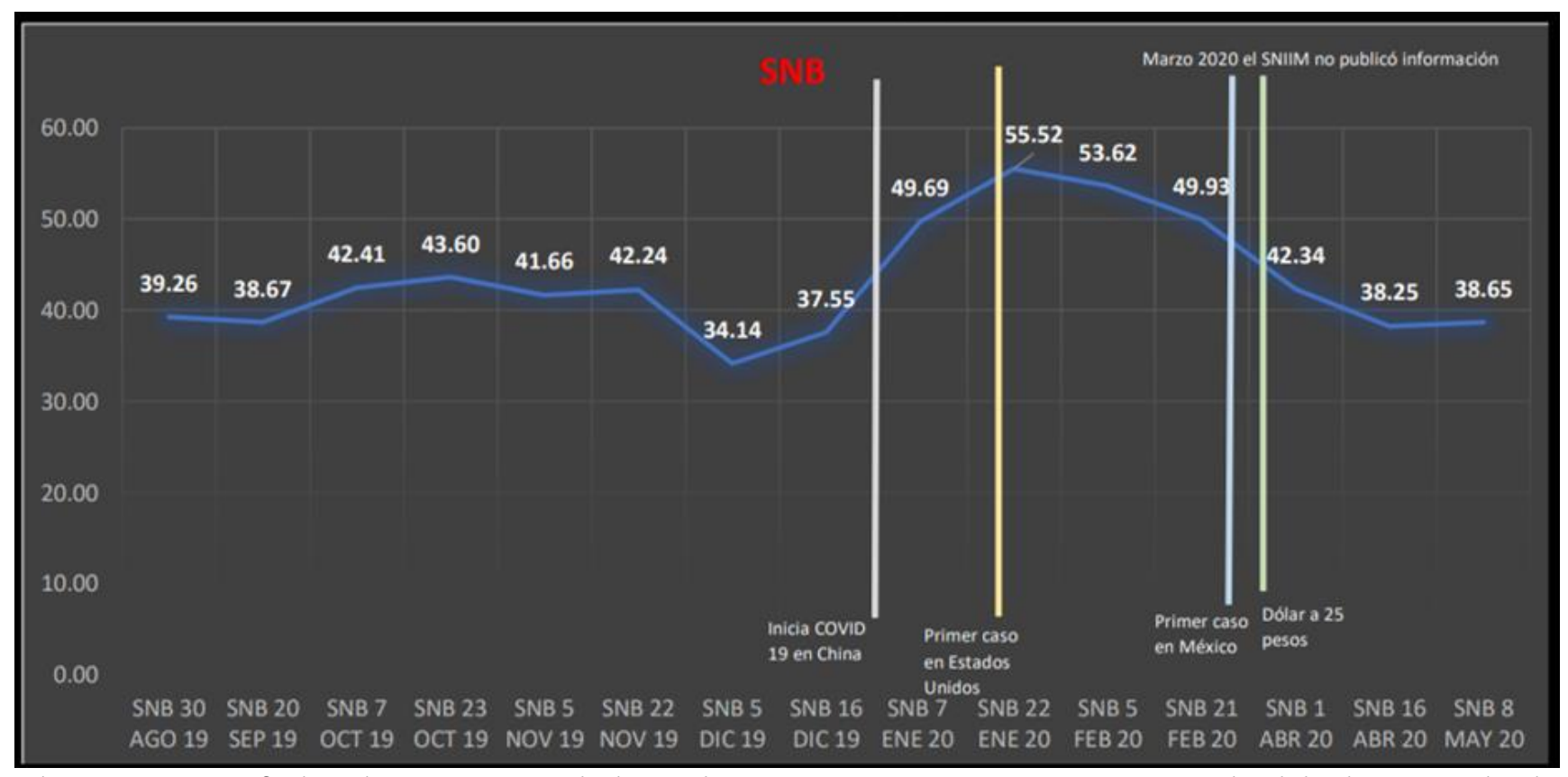

Figura 2. SNB y fechas importantes relativas al COVID-19. Fuente: Imagen capturada del Observatorio de Economía y Sociedad (OBSERVES); García. (2020b). Sustracción Nominal Básica (SNB). Boletín número especial Pandemia Covid-19 del día 14 de mayo de 2020. Autor: Alberto García Leyva. Disponible en: https://www.uv.mx/observes/files/2018/05/SNB-2020-especial-covid-19-2.pdf

En la imagen de la gráfica se observa que la SNB comienza a disminuir a partir de que se presentan los primeros casos de COVID-19 en los Estados Unidos. Esto puede o no tener relación; de tenerla, se puede deber a un aumento en el precio de algún insumo necesario para la producción de los alimentos provenientes de este país, para la fecha del primer caso en México de Covid-19 y del dólar a 25 pesos que se marca en la gráfica, se observa igualmente una disminución de la SNB pero pareciera más un efecto de inercia que de una consecuencia de los hechos ocurridos en ese periodo; independiente del caso, es necesario un estudio más detallado sobre la correlación de los eventos y el comportamiento de la SNB. También es importante mencionar que durante este periodo el BANXICO ha disminuido las tasas de interés, lo que igualmente pudo o no haber tenido una reacción en los precios locales.

\section{La PSHD y su comportamiento}

Otro de los indicadores monitoreados por el OBSERVES es la PSHD, el cual mide el gasto en materia de consumo de agua que una sola persona tiene de manera doméstica, este consumo/gasto es medido en proporción de un salario mínimo mensual vigente, se calcula para la ciudad de Xalapa tomando en cuenta el precio de la tarifa de servicio de agua por metro 
cúbico, así que se toma la información proveniente de la Comisión Municipal de Agua potable y Saneamiento de Xalapa Veracruz para considerar el precio del agua, se puede tomar una ponderación o precios máximos y mínimos según la información que se utilice de los rangos de precios publicados por la fuente primaria, por lo que las cantidades tomadas en cuenta pueden ser variadas dependiendo lo que se requiera analizar, se trata de un indicador con características adaptativas, según convenga para la investigación. En general, el indicador está diseñado para ser adaptado a cualquier ciudad, si se cuenta con la información disponible, lo que permite la posibilidad de ser calculado para diversas ciudades, salarios, consumos, precios, espacios temporales y comparar resultados (García, 2018b).

La evolución y el comportamiento de este indicador, a través del tiempo, son monitoreados por el OBSERVES y los resultados son publicados a manera de boletines informativos; estos pueden ser consultados por el público en general (OBSERVES, S.F.b). los boletines se publican desde el mes de julio del 2018 y tiene una publicación mensual e ininterrumpida para mayo del 2020, último boletín a fecha de elaboración de este artículo de investigación.

El boletín número 23 correspondiente al mes de mayo del año 2020 (OBSERVES; García, 2020c), muestra, en una primera parte, el resumen de la metodología con la cual se determinó el resultado, así como la fórmula de la PSHD; se explica que los resultados están conformados de dos formas: la primera se trata de un porcentaje para precios en zona popular denominada (PSHD-) y la segunda estimación con precios para zona residencial, denominada (PSHD+), de esta manera se exhibe los dos rangos máximos y mínimos de la proporción para tarifa de agua de 0-10 metros cúbicos, éstos son los rangos de precios del agua que una persona paga por un mes comparado con un salario mínimo mensual. Posteriormente, se muestra el resultado del salario mínimo vigente, los precios de la tarifa del agua con su correspondiente fuente de información primaria, la Comisión Nacional de los Salarios Mínimos y la CMAS Xalapa en orden y, finalmente, se muestra el resultado de la (PSHD -) y la (PSHD+), que para ese mes fue de $1.68 \%$ y $3.60 \%$ respectivamente, para incluir una parte final del boletín con graficas de la evolución de los resultados. 


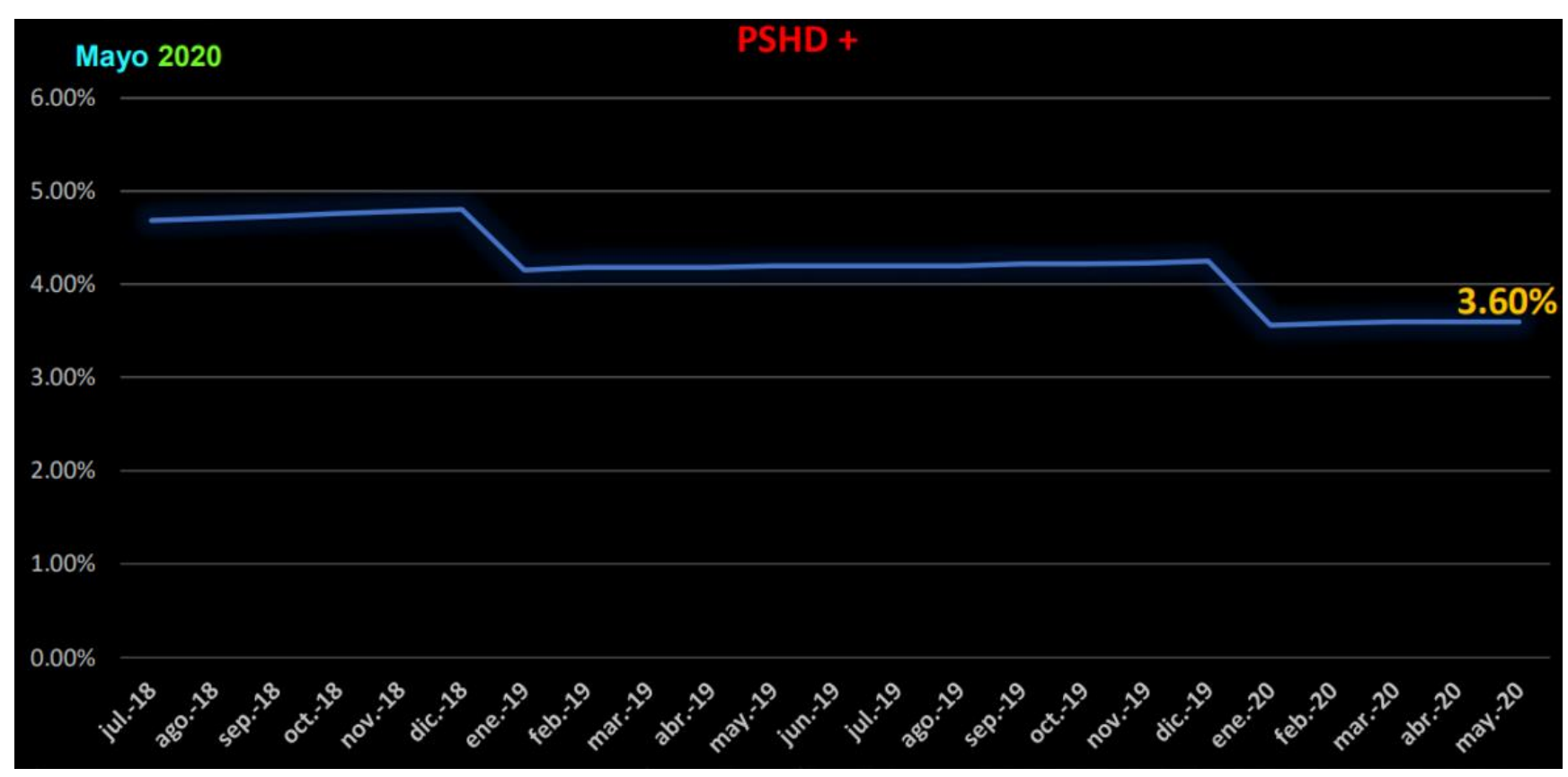

Figura 3. (PSHD +) Julio 2018- mayo 2020. Fuente: Imagen capturada del Observatorio de Economía y Sociedad (OBSERVES); García. (2020c). Proporción Salarial Hídrica Doméstica (PSHD). Boletín número 23 del mes de mayo de 2020. Autor: Alberto García Leyva. Disponible en: https://www.uv.mx/observes/files/2018/08/PSHDMAYO-2020.pdf

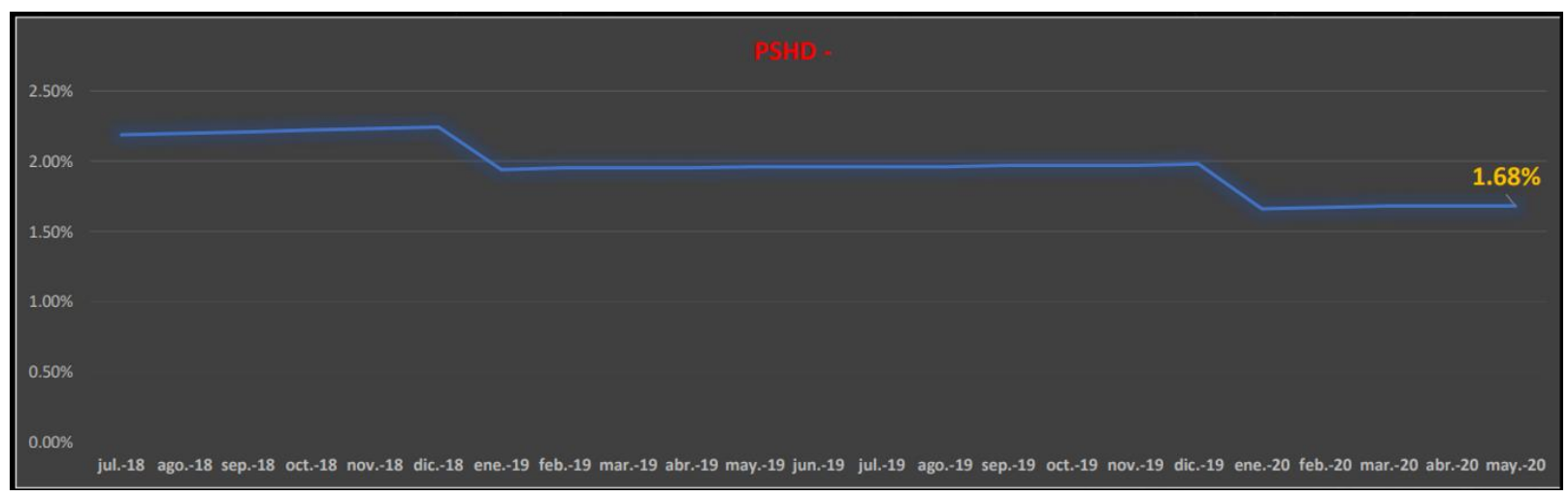

Figura 4. (PSHD -) Julio 2018- mayo 2020. Fuente: Imagen capturada del Observatorio de Economía y Sociedad (OBSERVES); García. (2020c). Proporción Salarial Hídrica Doméstica (PSHD). Boletín número 23 del mes de mayo de 2020. Autor: Alberto García Leyva. Disponible en:

https://www.uv.mx/observes/files/2018/08/PSHD-MAYO-2020.pdf

En las figuras anteriores, publicadas en el boletín de mayo del 2020, se puede observar la evolución de los dos rangos de la PSHD para los meses comprendidos de entre julio de 2018 a mayo de 2020, con una tendencia negativa, motivada por el aumento al salario mínimo que se presenta cada año, después de las reducciones de estos cambios, se observa un ligero aumento propiciado por el aumento en el precio de las tarifas del agua, a diferencia de la SNB, antes expuesta; la PSHD se comporta directa a los precios establecidos, por tal motivo se observa un 
comportamiento más regulado y no tan oscilante. Es importante señalar la necesidad de elaborar análisis e investigaciones en torno al precio del agua en términos reales, sin tomar en cuenta la inflación, para visualizar cómo ha variado el precio de consumo de agua personal domestica a través del tiempo.

\section{Conclusión}

La información que se presenta típicamente en el OBSERVES, corresponde a indicadores con metodologías originales, que versan sobre conocer las condiciones en las que la población se desarrolla de manera local, aunque la contribución también se puede adaptar a las diferentes ciudades del país e inclusive del mundo.

Se trata de generar información que pueda ser utilizada para los diferentes sectores de la sociedad interesados en el tema, es una contribución que se realiza para ayudar a mejorar la información que aborda la problemática en las que se desarrolla una persona y una sociedad, es también un esfuerzo para capturar en números, de fácil interpretación, realidades sumamente complejas y difíciles de abordar, que en muchas ocasiones por falta de información pertinente no son atendidas.

Los indicadores expuestos en OBSERVES tienen la peculiaridad de estar enfocados en la persona; es decir, en un elemento individual de la sociedad, ya que lo que influye en el comportamiento de un individuo son los fenómenos agregados sociales, y a su vez es el sentir y condiciones que una persona tiene las que tendrán un reflejo en el comportamiento general de una sociedad. De ahí la importancia de que las políticas públicas estén enfocadas también en la persona, en mejorar sus condiciones de vida, porque a su vez tendrán una respuesta de manera agregada, esto concluye con la necesidad de generar información enfocada a la realidad que experimenta un sujeto desde su individualidad.

\section{Referencias}

García Leyva, Alberto. (2018a). Indicador de precios, gastos y poder adquisitivo nominal entorno a un consumo alimenticio saludable. Una propuesta desde el Observatorio de Economía y Sociedad de la Universidad Veracruzana. UVServa. Revista electrónica de la Coordinación Universitaria de Observatorios, (5), 85-90. 
García Leyva. (2018b). Proporción Salarial Hídrica Doméstica, propuesta de indicador entorno al gasto básico personal. Interconectando Saberes, 3(5), 75-85. Recuperado de: http://is.uv.mx/index.php/IS/article/view/2549/4466

Observatorio de Economía y Sociedad (OBSERVES). (S.F.a). Sustracción Nominal Básica (SNB). Boletines. Recuperado de: https://www.uv.mx/observes/general/sustraccion-nominalbasica-snb/

Observatorio de Economía y Sociedad (OBSERVES). (S.F.b). Proporción Salarial Hídrica Doméstica (PSHD). Boletines. Recuperado de:

https://www.uv.mx/observes/general/proporcion-salarial-hidrica-domestica-pshd/

Observatorio de Economía y Sociedad (OBSERVES); García. (2020a). Sustracción Nominal Básica (SNB). Boletín número 53 del día 22 de mayo del 2020. Autor: Alberto García Leyva. Recuperado de: https://www.uv.mx/observes/files/2018/05/SNB-2020-53-1.pdf

Observatorio de Economía y Sociedad (OBSERVES); García. (2020b). Sustracción Nominal Básica (SNB). Boletín número especial Pandemia Covid-19 del día 14 de mayo del 2020. Autor: Alberto García Leyva. Recuperado de: https://www.uv.mx/observes/files/2018/05/SNB-2020-especial-covid-19-2.pdf

Observatorio de Economía y Sociedad (OBSERVES); García. (2020c). Proporción Salarial Hídrica Doméstica (PSHD). Boletín número 23 del mes de mayo del 2020. Autor: Alberto García Leyva. Recuperado de: https://www.uv.mx/observes/files/2018/08/PSHD-MAYO2020.pdf 